\title{
Tibial Plateau Geometry Influences Lower Extremity Biomechanics During Landing
}

\author{
By: Sandra J. Shultz and Randy J. Schmitz
}

Shultz SJ, Schmitz RJ. Tibial Plateau Geometry Influences Hip and Knee Motion During Landing. American Journal of Sports Medicine 2012;40(9): 2029-2036.

\begin{abstract}
***@) The Authors. Reprinted with permission. No further reproduction is authorized without written permission from SAGE Publications. This version of the document is not the version of record. Figures and/or pictures may be missing from this format of the document. $* * *$
\end{abstract}

Made available courtesy of SAGE Publications: http://dx.doi.org/10.1177/0363546512453295.

Abstract:

Background: Intersubject differences in lateral and medial posterior tibial plateau slope, coronal tibial slope (CTS), and medial tibial plateau depth (MTD) may influence one's susceptibility for anterior cruciate ligament (ACL) injury. Understanding how these structural characteristics influence hip and knee joint biomechanics during weightbearing activity may advance our understanding of how tibial plateau geometry influences one's injury risk potential.

Purpose/Hypotheses: To determine the extent to which tibial plateau geometry is associated with frontal and transverse plane hip and knee joint biomechanics during the initial landing phase of a double-legged drop landing. Greater lateral tibial slope combined with lower medial/lateral tibial slope ratio would predict greater tibial internal rotation motion and moments. Lower CTS would predict greater hip adduction and knee valgus motion and reduced internal peak varus moments. These associations would be stronger when combined with a shallower MTD.

Study Design: Descriptive laboratory study.

Methods: Magnetic resonance scans of the knee were obtained on 23 female participants who were also assessed for hip and knee joint biomechanics during the initial landing phase of doublelegged drop jumps.

Results: Once controlling for the respective initial hip flexion or initial knee flexion angle at ground contact, lower CTS consistently predicted greater initial and peak hip adduction and knee valgus angles $\left(R^{2}\right.$ range, $\left..212-.427, P<.027\right)$. Greater coronal and lateral tibial slope predicted greater hip internal rotation (femur relative to pelvis) at initial contact $\left(R^{2}=.504\right)$ and greater CTS and lower medial/lateral tibial slope ratio predicted greater knee internal rotation (tibia relative to femur) excursions $\left(R^{2}=.594, P=.001\right)$. Joint geometry was not associated with hip or knee peak joint moments.

Conclusion: These data confirm substantial intersubject differences in tibial condylar geometry that are associated with intersubject differences in hip and knee motion patterns when landing from a jump.

Clinical Relevance: The current findings may partially explain a female's greater likelihood of demonstrating combined motion patterns of knee valgus and external rotation during landing. Although tibial geometry cannot be modified through training, these associations suggest that tibial 
geometry may have a substantial influence on tibiofemoral joint biomechanics when the knee is subjected to external loads and deserves further study in our understanding of ACL injury.

Keywords: tibial slope | tibial condylar depth | landing biomechanics

\section{Article:}

Increasing evidence shows that the shape and slope of the tibial plateau (posterior inferior inclinations of the medial [MTS] and lateral [LTS] tibial plateaus, coronal slope of the tibial plateau [CTS], and depth of the medial condyle concavity [MTD]) vary substantially among individuals $\underline{15}, \underline{30}, \underline{47}$ and can influence one's susceptibility for anterior cruciate ligament (ACL) injury. $\underline{3}, \underline{17}, \underline{20}, \underline{25}, \underline{46} \underline{48}, \underline{50}$ Case-control studies measuring tibial geometry from lateral-view plain radiographs (where the medial plateau is typically measured because it is easier to identify $\underline{48}$ ) reported greater MTS in ACL-injured cases compared with controls, $\underline{3}, \underline{48}, \underline{50}$ whereas another reported no difference. 34 Conversely, studies measuring tibial plateau geometry from magnetic resonance images (in which both MTS and LTS are well visualized) $\underline{17}, \underline{20}, \underline{25}, \underline{46}, \underline{47}$ report greater LTS (but not MTS) in ACL-injured versus control cases, $25, \underline{46}, \underline{47}$ whereas 1 study reported both greater LTS and MTS in male ACL-injured patients versus controls (but only greater LTS in female patients vs controls), 17 and another reported no difference in LTS or MTS between cases and controls.20 Collectively, these studies largely support a greater susceptibility for ACL injury when the posterior-inferior slope of the tibial plateau is increased. Furthermore, magnetic resonance imaging (MRI) studies suggest the LTS may better differentiate ACL-injured cases from controls than the MTS, which may imply that the relative magnitude of the LTS versus the MTS is also important. Although direct statistical comparisons are limited, mean LTS values are typically larger than MTS values in ACL-injured cohorts, regardless of the study, $15,20,25,46$ whereas mean LTS values are larger than MTS values in some control groups $15,20,46$ but lower in others.25 $\underline{30}, \underline{47}$ Only 2 studies have directly compared the relative magnitudes of the LTS versus the MTS in relation to injury status. Although 1 study reported a positive mean difference score (LTS > MTS) in ACL-injured cases that was significantly larger than the negative mean difference score (LTS $<$ MTS) in uninjured controls, $\underline{47}$ the other study reported no relative difference in the LTS versus MTS in cases versus controls.20 Less is known about MTD and CTS. Of 2 studies that have measured MTD, both reported a shallower depth in ACL-injured versus control cases. 17,25 Only 1 study measured CTS, and no difference was found between cases and controls. 17

Although these tibial plateau characteristics cannot be modified, understanding their influence on tibiofemoral joint biomechanics when the knee is subjected to external loads (eg, those produced by weightbearing activities, such as landing from a jump) or internal loads (eg, compressive and shear forces created by the thigh muscles) may allow us to appropriately account for these risk factors in our ACL prevention strategies. To that end, prior research indicates that greater posterior-inferior tibial slopes are associated with greater anterior joint reaction force $\underline{33}$ and greater anterior translation of the tibia relative to the femur $\underline{5} 13$ during axial loading. More recently, it has been theorized that high-risk knee motions in the transverse and frontal plane may also be influenced by these tibial plateau geometries. Simon et al $\underline{46}$ posited that the relatively flat surface and greater slope of the LTS relative to the MTS may promote greater anterior translation of the lateral versus medial tibia plateau, $\underline{33} \underline{46}$ resulting in concomitant internal 
rotation of the tibia relative to the femur, coupled motions known to strain the ACL. $\underline{41}$ In addition, a deeper MTD may be associated with greater restraint of the medial femoral condyle, resulting in decreased tibiofemoral displacements. 15 In the coronal plane, it has been theorized that a lower CTS (medial plateau contact more superior than the lateral plateau contact) may also influence knee biomechanics and ACL strain by promoting greater dynamic knee valgus. 16 As females have greater MTS and LTS inclinations $\underline{15} \underline{20}$ and reduced CTS inclinations compared with males, 15 these theories lead to speculation that sex differences in tibial plateau geometry may in part explain a female's greater susceptibility to knee motions during landing and plantand-cut maneuvers that are considered to put them at higher risk for ACL injury. 14, 19, $\underline{35}, \underline{37}, \underline{40}$

We are aware of only 1 in vivo study that has examined relationships between tibial plateau characteristics and high-risk transverse and frontal plane lower extremity biomechanics during weightbearing activity. 33 During a single-legged land-and-cut task, McLean et al 33 reported a strong association between lower MTS:LTS ratios (lateral slope > medial slope) and greater peak knee valgus and internal rotation of the tibia relative to the femur. However, CTS and MTD were not evaluated, and associations were limited to the knee. Our purpose was to build on this research and determine the extent to which tibial plateau geometries commonly associated with ACL injury (LTS, MTS:LTS, and MTD) or otherwise reported to differ in males and females (CTS) influence frontal and transverse plane hip and knee biomechanics during the initial landing phase of a double-legged drop landing. Consistent with the theories set forth, we hypothesized that greater LTS combined with a lower MTS:LTS ratio would be associated with greater internal rotation motion and moments of the tibia relative to the femur and that lower CTS would be associated with greater hip adduction and knee valgus motion and reduced internal peak varus moments (resisting external knee valgus moment). We further expected these associations would be stronger when combined with a shallow MTD.

\section{METHODS}

Magnetic resonance imaging scans of the knee were obtained on 23 female volunteers (age, $21 \pm$ 2.8 years; height, $165.7 \pm 7.5 \mathrm{~cm}$; weight, $63.8 \pm 10.7 \mathrm{~kg}$ ) who participated in a larger study that included assessment of their hip and knee biomechanics during the initial landing of a doublelegged drop jump. $43 \Downarrow-45$ All were physically active (2-10 h/wk of physical activity) and had no history of orthopaedic lower extremity injury, as confirmed by a health history questionnaire. The study was approved by the university's institutional review board, and all volunteers provided written consent to participate. All measurements were obtained on the dominant-stance limb (defined as the preferred-stance limb when kicking a ball), and biomechanical testing was restricted to the first 6 days of the menstrual cycle to control for potential influences of hormone concentration changes on joint neuromechanics.

\section{Tibial Geometry}

T1-weighted, multiplanar MRI scans $(1.5 \mathrm{~T}$; voxel size, $0.55 \times 0.55 \times 0.5 \mathrm{~mm})$ were obtained from the dominant knee. Using methods described by Hashemi et al, $\underline{15}$ we measured tibial condylar geometry using the Medical Image Processing, Analysis and Visualization software (MIPAV; http://mipav.cit.nih.gov). To determine the sagittal and coronal slices from which to measure MTS, LTS, CTS, and MTD, the transverse plane image that showed the maximal tibial 
plateau area was identified (Figure 1). From this image, the sagittal plane slice that bisected the medial plateau at the point of greatest anterior to posterior distance was selected to measure MTS (and MTD), and the sagittal slice that bisected the lateral plateau at the point of greatest anterior to posterior distance was selected to measure the LTS.15 The coronal slice nearest to the anterior/posterior centroid of the tibial plateau was selected to measure CTS.

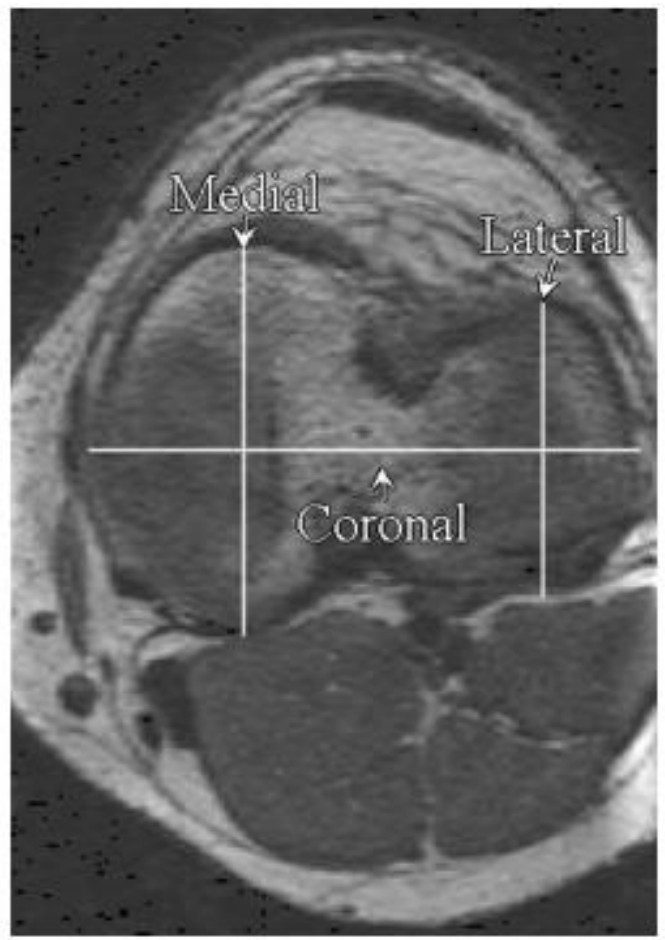

Figure 1. Orientation of magnetic resonance imaging slices obtained for tibial geometry measures.

The MTS and LTS were measured as the angle between a line connecting the peak anterior and posterior aspects of the medial and lateral tibial plateaus, respectively, and a line representing the longitudinal axis of the tibia (Figure 2, A and B). The longitudinal axis of the tibia was estimated using a sagittal plane image aligned near the lateral margin of the tibial plateau that clearly showed the orientation of the tibia. We then identified the center point of the tibial cortex at $5 \mathrm{~cm}$ and $10 \mathrm{~cm}$ distal to the tibial plateau and then drew a vertical line connecting these 2 points. This vertical line representing the axis of the femur was then transposed to the sagittal images from which the MTS and LTS measures were obtained. If the posterior plateau contact point was inferior to the anterior plateau contact point, the slope was positive.

The CTS was measured as the angle between a line joining the peak points of the medial and lateral tibial plateaus and a line perpendicular to the longitudinal axis of the tibia (Figure 2C). Using the image from the transverse plane slice (Figure 1), a line was drawn connecting the peak points of the medial and lateral tibial plateaus. The longitudinal axis of the tibia was estimated by drawing a vertical line that connected the centroids of the cortex at distances of $5 \mathrm{~cm}$ and $10 \mathrm{~cm}$ distal to the tibial plateau. If the medial plateau contact point was positioned inferior to lateral plateau contact point, the slope was positive. 


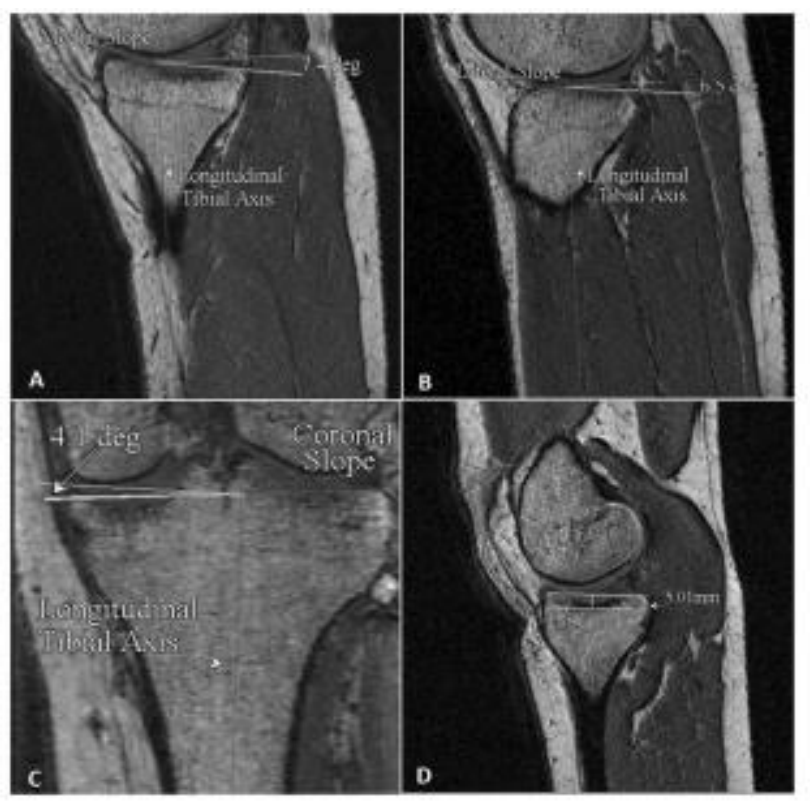

Figure 2. Measurement of (A) medial posterior-inferior tibial slope, (B) lateral posterior-inferior tibial slope, (C) coronal tibial slope, and (D) medial condylar depth.

Medial condylar depth was measured from the same sagittal plane slice as the MTS (Figure 1). The MTD was measured in millimeters as the perpendicular distance between a line drawn between the anterior and posterior tibial plateau crests and a parallel line inserted tangentially to the most inferior point of medial tibial body (Figure 2D).

A single investigator (R.J.S.) obtained 3 separate measurements for each variable, and the average was used for the analyses. Before the study, excellent test-retest measurement of tibial geometry was confirmed by taking measurements on 2 separate occasions (24-48 hours apart) from the images of 10 participants (see Appendix Table A1, available in the online version of this article at http://ajs.sagepub.com/supplemental/).

\section{Landing Biomechanics}

Kinematic data for the dominant limb foot, shank, thigh, pelvis, and trunk were collected at 100 $\mathrm{Hz}$ using an electromagnetic tracking system (Motion Star; Ascension Technologies, Burlington, Vermont) as previously described. 42 Briefly, position sensors were attached to each participant's dominant limb over the midshaft of the anterior surface of the third metatarsal, the medial tibia, and the lateral aspect of the femur. Additional sensors were placed on the contralateral femur and tibia, on the sacrum, and over the $\mathrm{C} 7$ spinous process. Vertical ground-reaction forces were obtained $(1000 \mathrm{~Hz})$ with a nonconducting force plate (Type 4060; Bertec Corp, Columbus, Ohio). Once fully instrumented, participants performed bilateral, barefooted drop-jump landings from a wooden platform, measuring $0.45 \mathrm{~m}$ in height and placed $0.1 \mathrm{~m}$ behind the rear edge of the force plate.42 For all landings, participants began with their hands held at shoulder level and palms facing forward, with their toes aligned along the leading edge of the platform. Participants were instructed to drop down, land evenly on both feet (dominant foot centered on the force plate; nondominant foot completely off the plate), and immediately perform a maximal-effort 
double-legged vertical jump upon landing. Participants were not given special landing instructions to prevent experimenter bias. After 3 to 6 practice repetitions, 5 drop-jump landing trials were recorded for analyses.

Kinetic (forceplate) data were offline low-pass filtered at $12 \mathrm{~Hz}$ using a fourth-order, zero-lag Butterworth filter. Hip joint centers were calculated using the Leardini method.27 Knee and ankle joint centers were calculated as the centroid of the medial and lateral femoral epicondyles and the medial and lateral malleoli, respectively.28 A segmental reference system defined all body segments with the positive z-axis as the left to right axis, the positive y-axis as the distal to proximal longitudinal axis, and the positive $\mathrm{x}$-axis as the posterior to anterior axis. Hip, knee, and ankle flexion angles were calculated using Euler angle definitions with a rotational sequence of $\mathrm{Z} \mathrm{Y}^{\prime} \mathrm{X}^{\prime \prime} . \underline{23}$ Raw kinematic data were linearly interpolated to force plate data and subsequently low-pass filtered at $12 \mathrm{~Hz}$ using a fourth-order, zero-lag Butterworth filter.

The initial landing phase was defined as the interval from the time of initial ground contact (ground-reaction force exceeding $10 \mathrm{~N}$ ) to the time when the body's center of mass reached its lowest position. Frontal and transverse plane hip and knee angles were acquired for initial contact, peak values, and total excursions (peak - initial joint angle) and averaged across the 5 landing trials for analysis. Frontal and transverse plane hip and knee intersegmental peak moments were calculated using inverse dynamics 12 (Motion Monitor Software; Innovative Sports Training, Chicago, Illinois) and normalized to the product of body weight and height $\left(\mathrm{Nm}^{*} \mathrm{BW}^{-} \underline{1} * \mathrm{Ht}^{-} \underline{1}\right)$. Positive/negative values were assigned for hip abduction $(+) /$ adduction $(-)$, hip external $(+)$ /internal $(-)$, knee valgus $(+)$ /varus $(-)$, and knee external $(+)$ /internal $(-)$ rotation motions and moments. Biomechanical data obtained from 50 male participants measured on 2 occasions approximately 2 weeks apart confirmed our ability to obtain relatively stable kinematic and kinetic data during this landing task (intraclass correlations coefficients $\left[\mathrm{ICC}_{2,5}\right]$ and standard error of measurements [SEM] are reported in Appendix Table A1, and 95\% limits of agreement and Bland-Altman plots are provided in Appendix Figure A1, available online).

\section{Statistical Analyses}

Backward stepwise multiple linear regression analyses were used to determine the extent to which tibial plateau geometry (LTS, MTS:LTS, CTS, and MTD) predicted the primary transverse and frontal plane hip and knee kinematics: hip adduction and internal rotation (referenced as femoral motion relative to the pelvis), knee valgus and internal rotation (referenced as tibial motion relative to the femur), and moments (hip adduction and external rotation; knee varus and internal rotation) observed during the initial landing phase of a dropjump task. 43 For each model, we also entered the initial hip (when predicting hip kinematics or kinetics) or knee (when predicting knee kinematics or kinetics) flexion angles to control for the initial femoroacetabular and tibiofemoral contact positions at ground contact. Our rationale for including these variables is based on a report that the initial knee flexion angle can influence subsequent coupled knee motions that occur during weightbearing. 22 All variables were entered simultaneously to determine the independent effects of each variable while controlling for the others. We then used a backward stepwise elimination $(P$ value removal tolerance $>.10)$ to determine if the prediction model could be simplified to fewer variables without substantially reducing the variance explained. $\underline{38}$ The $\alpha$ level for all analyses was set at $P<.05$. Power to 
detect a significant multiple $R^{2}$ of 0.35 or higher (ie, large effect) with 23 participants was estimated to range from $70 \%$ to $90 \%$ depending on the number of predictors (1-4) that remained in the final model. $\underline{8}$

\section{RESULTS}

The descriptive data for each predictor and dependent variable are presented in Appendix Table A1 (available online). The final regression summary results for the extent to which tibial plateau geometry predicted hip and knee transverse and frontal plane biomechanics are presented in Appendix Table A2 (available online).

In the frontal plane, women with lower CTS inclinations (medial more superior than the lateral plateau contact) were more likely to initially land in greater hip adduction $\left(R^{2}=.413, P=.001\right)$ and knee valgus $\left(R^{2}=.221, P=.024\right)$. Lower CTS was also a predictor of peak hip adduction $\left(R^{2}=.427, P=.001\right)$ and knee valgus $\left(R^{2}=.212, P=.027\right)$ but not hip adduction or knee valgus excursion values. Based on the final regression models, for every $1.0^{\circ}$ decrease in CTS, there was a predicted increase of $1.2^{\circ}$ and $1.8^{\circ}$, in initial and peak hip adduction angles, and a predicted increase of $0.7^{\circ}$ and $1.2^{\circ}$, in initial and peak knee valgus angles, respectively (see Appendix Table A2). Hence, those with lower CTS began in more hip adduction and knee valgus and remained in more hip adduction and knee valgus throughout the landing. This is confirmed by Figure 3, which graphically depicts these motions over the entire landing phase, with women stratified into below-average $(\mathrm{n}=10)$ and above-average $(\mathrm{n}=13)$ CTS groups.
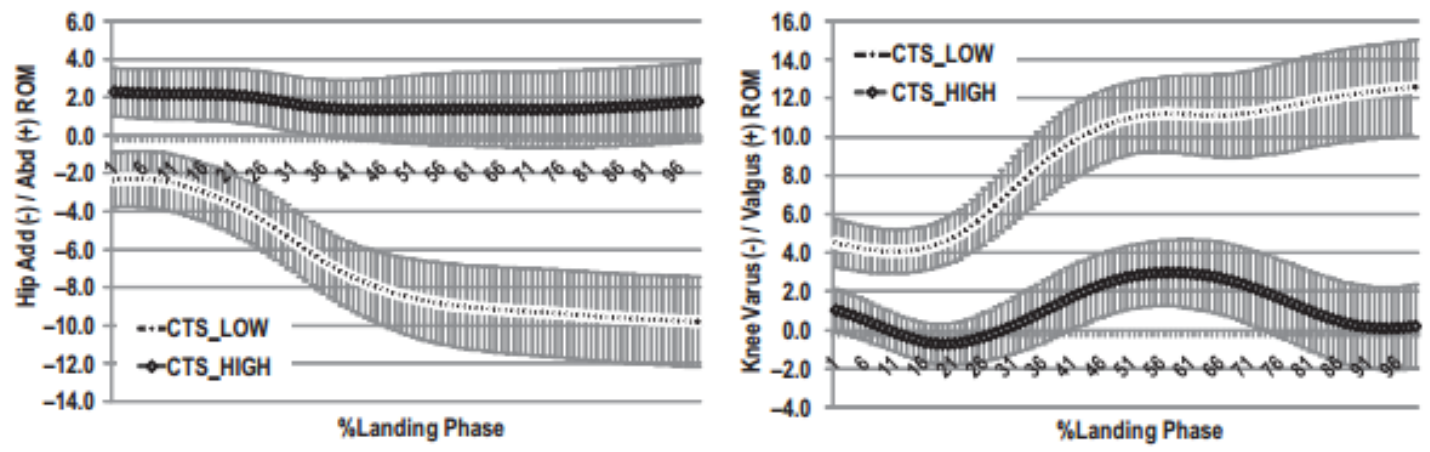

Figure 3. Comparative hip adduction and knee valgus angles (mean \pm standard error) during the initial landing phase of a drop jump in women with below-average $\left(\leq 4.0^{\circ}\right)\left(1.4^{\circ} \pm 1.8^{\circ} ; n=10\right)$ and above-average $\left(>4.0^{\circ}\right)\left(6.1^{\circ} \pm 1.1^{\circ} ; n=13\right)$ coronal tibial slope (CTS) angles. Abd, abduction; Add, adduction; CTS, coronal tibial slope; ROM, range of motion.

In the transverse plane, female participants with higher LTS and higher CTS were more likely to land in greater hip internal rotation at initial contact $\left(R^{2}=.504, P=.003\right)$; however, joint geometry was not a significant predictor of peak or total excursion of hip internal rotation. At the knee, knee joint geometry was not a predictor of initial or peak knee internal rotation angles. However, female participants with lower MTS:LTS ratios (MTS<LTS), greater CTS inclinations, and a more extended knee upon ground contact were more likely to go through greater knee internal rotation excursions $\left(R^{2}=.594, P=.001\right)$ (see Figure 4$)$. For every 0.1 decrease in the MTS:LTS ratio and $1^{\circ}$ increase in CTS, there was a $2.2^{\circ}$ increase in knee internal rotation excursion. Tibial plateau geometry was not a significant predictor of hip adduction, hip external rotation, knee varus, or knee internal rotation internal peak joint moments $\left(R^{2}\right.$ change once accounting for hip or knee flexion angle $=.105-.202 ; P>.384$ ). 


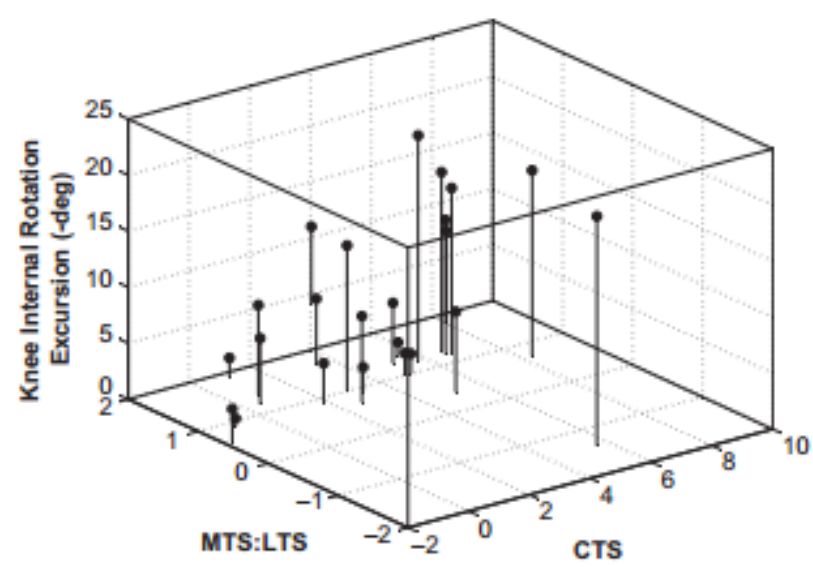

Figure 4. Three-dimensional scatterplot depicting the relationship between lower MTS:LTS ratio and higher CTS predicting greater knee internal rotation angle. Note: Decreased knee flexion angle is not included in this graphic representation, but it was also a significant predictor of greater knee internal rotation excursion. CTS, coronal tibial slope; LTS, lateral posterior-inferior tibial slope; MTS, medial posterior-inferior tibial slope.

\section{DISCUSSION}

Numerous investigations have examined the relationship between tibial condylar geometry and ACL injury risk. However, few have examined the consequences of these tibial condylar geometries on weightbearing biomechanics, which may provide a better understanding of their potential role in the ACL injury mechanism. Our primary findings indicate that the LTS, MTS:LTS, and CTS are associated with transverse and frontal plane hip and knee motions (but not moments) during the initial landing phase of a drop jump, whereas the MTD had no relationship with hip or knee joint biomechanics.

Comparing the magnitude and range of our tibial plateau measures with prior research suggests our values are well in line with those reported for LTS 15,2025 and MTD $\underline{15}, \underline{25}$ in uninjured individuals. Among the few prior reports of MTS:LTS and CTS values, our MTS:LTS (1.1 \pm 0.8 vs $0.7 \pm 0.1 \underline{33})$ and $\operatorname{CTS}\left(4.1^{\circ} \pm 2.8^{\circ}\right.$ vs $2.5^{\circ} \pm 1.9^{\circ} \underline{15}$; range, $-1.8^{\circ}$ to $8.3^{\circ}$ vs $0.0^{\circ}$ to $\left.6.0^{\circ} \underline{15}\right)$ values were somewhat larger and more variable.

Our hypotheses regarding frontal plane hip and knee kinematics were largely supported in that lower CTS inclinations were consistent predictors of greater initial and peak hip adduction and knee valgus angles. Our hypothesis was based on the theory that a more inferior tibiofemoral contact point for the lateral versus medial tibial plateau (ie, lower CTS) would result in relatively greater valgus angulation 16 and a concomitant increase in hip adduction. The coupled increase in both hip adduction and knee valgus (Pearson correlations of .542, .623, and .669 for initial, peak, and excursion values) is consistent with studies reporting both greater peak hip adduction and knee abduction angles in female runners compared with male runners $\underline{9}$ and a strong association between hip adduction and knee valgus motion at the time of peak force during the stance phase of cutting maneuvers.21 Although the latter study attributed this coupling to reduced hip 
abduction strength, 21 our findings suggest that knee anatomy (CTS) could also be a driving factor.

The comparative hip adduction and knee valgus angles in female participants with above- versus below-average CTS (Figure 3) suggest that the intersubject variability in CTS among females $\left(-1.8^{\circ}\right.$ to $\left.8.3^{\circ}\right)$ may have a meaningful effect on frontal plane hip and knee motions, as the magnitude of peak differences well exceeds what would be expected simply due to measurement error (see supplementary data in the online Appendix). Although CTS has only been examined relative to ACL injury risk in 1 study and did not discriminate between cases and controls, 17 there is 1 report of female participants having lower CTS values compared with males.15 Based on our data, lower CTS in females would be consistent with the greater hip adduction and knee valgus angles observed in females versus males during running, landing, and cutting maneuvers. $9 \Downarrow 11,18,24,29,31$ Although additional studies are needed, these findings suggest that the lower CTS reported in females compared with males 15 may in part explain a female's greater susceptibility to knee motions during landing and plant-and-cut maneuvers that are commonly thought to put them at risk for ACL injury.14, 19, $\underline{35}, \underline{30}$ However, it should be noted that these kinematic associations with tibial plateau geometry were not accompanied by significant associations with frontal plane hip and knee moments. That is, even though females with lower CTS demonstrated substantially more knee valgus, tibial geometry had no effect on the internal varus moments that would act to resist external loads acting on the bony orientation. It may be that these females have adapted to this anatomic predisposition of increased valgus angle (with a corresponding greater frontal plane moment arm from the knee joint center to the mechanical hip to the ankle joint center axis) by altering their global movement patterns to control valgus loading about the knee during a controlled drop landing. This potential explanation is supported by research documenting that valgus loading during impact activities can be decreased through proximal technique changes. 6 It may also be that the intersubject differences observed within this healthy female population, although substantial, simply reflect a normal range of anatomic variations that alone are not sufficient to adversely affect frontal plane knee loads during this controlled task. Whether similar findings would be observed during unplanned tasks, less stable single-legged landings, or other tasks that need greater resistance to frontal plane moments requires further study.

When examining transverse plane hip and knee motions, our hypothesis that greater LTS coupled with lower MTS:LTS ratios would be associated with greater knee internal rotation motion and moments was only partially supported. Associations were limited to knee internal rotation excursion, where lower MTS:LTS (reduced MTS relative to LTS slope) predicted greater knee internal rotation during the initial landing phase if females also had greater CTS and a more extended knee. To appreciate the magnitude of this effect, we compared knee internal rotation motion patterns in the 10 female participants who had MTS:LTS ratios less than 1.0 that were further stratified into below-average and above-average CTS groups; those with above-average CTS values went through $6.2^{\circ}$ greater knee internal rotation during the landing than those with below-average CTS $\left(9.6^{\circ} \pm 8.6^{\circ}\right.$ vs $\left.3.4^{\circ} \pm 2.6^{\circ}\right)$. The magnitude of this mean difference in healthy individuals represents an effect size conservatively estimated at .72, which nears but does not exceed the $95 \%$ confidence limits of what would be expected simply due to measurement error (see Appendix, available online). Although the observed association between lower MTS:LTS and greater knee internal rotation is consistent with the theory by Simon et 
al, $\underline{46}$ the relationship we observed was substantially weaker than that reported by McLean et al $\underline{3}$ during a single-legged land-and-cut task. Specifically, for a 0.1 decrease in the MTS:LTS ratio, McLean et al reported a predicted 3.7 increase in peak knee internal rotation angle, whereas we predicted only a $0.8^{\circ}$ increase in knee internal rotation excursion (and no association with peak knee internal rotation angle). This may suggest the observed associations are relatively small during a controlled vertical double-legged drop-landing task but may be accentuated in a single-legged land-and-cut task. In addition, we included initial contact knee flexion angle and CTS in our prediction model, which could account for some of the unexplained variance in knee internal rotation excursion in the weightbearing knee.22 Although including these variables improved the independent correlation between MTS:LTS and knee internal rotation (zero-order correlation $=.326$ vs part correlation $=.523$ ), they were independent meaningful predictors of knee internal rotation (see Appendix Table 2, available online); for every $1^{\circ}$ decrease in initial knee flexion angle, there was a $0.4^{\circ}$ increase in knee internal rotation excursion, and for every $1^{\circ}$ increase in CTS, there was a predicted $1.4^{\circ}$ increase in knee internal rotation.

Another clear difference in McLean et al $\underline{33}$ study versus our study is that their female cohort had higher mean LTS magnitudes $\left(8.3^{\circ} \pm 1.7^{\circ}\right.$ vs $5.4^{\circ} \pm 2.6^{\circ}$, respectively) and similar MTS magnitudes $\left(5.7^{\circ} \pm 1.4^{\circ}\right.$ vs $5.8^{\circ} \pm 3.4^{\circ}$, respectively) and thus lower average MTS:LTS ratios $(0.7 \pm 0.1$ vs $1.1 \pm 0.8)$ than our female cohort. As the magnitude of anterior tibial translation is largely dependent on the absolute magnitude of the posterior-inferior slope, $\underline{5}, \underline{13}$ a critical magnitude of LTS may be necessary before the MTS:LTS ratio has a substantial effect on the relative translation of the lateral versus tibial plateau and thus knee internal rotation.

We did not observe the same coupling between the hip and knee for initial, peak, and excursion values in the transverse plane (Pearson $r=.235, .085$, and .188 , respectively) as we observed for the frontal plane. Rather, higher CTS and LTS were associated with more hip internal rotation at ground contact when the hip was more extended. Although conjecture at this point, it is possible that the body is attempting to initially land in greater hip internal rotation to account for a relatively greater expected external rotation of the femur on a fixed tibia (a resultant internal rotation at the knee joint) that may occur upon weightbearing in those with a greater LTS. Alternatively, the greater hip internal rotation at contact could be the result of an anticipation of potentially larger external rotation torque acting internally on the femur based on past experience, with the resultant development of a neuromuscular strategy based on such experiences. As with the frontal plane, these kinematic associations with tibial geometry were not accompanied by similar influences on transverse plane hip and knee moments. This again suggests that the range of hip and knee motions observed in these females reflects normal intersubject anatomic variations or that they are able to adequately adapt/compensate for these greater motions during this controlled landing task. Hence, these associations should be further investigated during more challenging landing maneuvers.

We did not observe any significant associations with MTD. This was surprising as a shallow MTD has been associated with ACL injury risk in 2 studies, $\underline{17}, \underline{25}$ with one reporting a 3 times greater risk per 1-mm decrease in MTD.17 It may be that the effects of MTD are largely limited to the anterior-posterior plane as theorized17,25or that the bilateral drop vertical jump used in the current study did not create a situation where the tibiofemoral shear component was sufficient to reach a threshold of potential subluxation or perceived subluxation of the tibia relative to the 
femur. Whether stronger associations with MTD and transverse and frontal plane knee motion would be observed during tasks that produce larger tibiofemoral shear loads requires further study. It may also be that our study sample of uninjured female participants may not have sufficiently represented the intersubject variability in the population. Although our mean MTD $(2.6 \pm 0.5 \mathrm{~mm})$ was quite comparable with that of others $(1.9 \pm 0.6 \mathrm{~mm} \underline{25}$ and $2.8 \pm 0.8 \mathrm{~mm} \underline{15})$, our range in values tended to be smaller (1.9-3.7 as compared with 0.1-3.52 $\underline{25}$ and 1.2-5.215), thus potentially limiting our ability to identify clear relationships.

In summary, our data confirm previous reports of substantial intersubject differences in tibial condylar geometry $15, \underline{30}$ that are associated with substantial intersubject differences in frontal and transverse plane lower extremity joint motions.33 Video analysis of ACL injury mechanisms describes knee motion patterns of knee valgus coupled with either knee internal or external rotation. $1,26,36$ Our findings revealed that lower CTS (a more inferior tibiofemoral contact point for the lateral vs medial tibial plateau) is associated with greater initial and peak hip adduction and knee valgus angles in females. Furthermore, lower CTS, when combined with a lower LTS and more extended hip, is associated with less initial hip internal rotation and, when combined with a higher relative slope of the MTS versus LTS and a more extended knee, is associated with less knee internal rotation excursions throughout the landing phase. However, within the constraints of our controlled double-legged vertical drop-landing task, we did not observe concomitant associations between tibial plateau geometry and hip and knee joint kinetics. Although CTS has not been found to differentiate ACL-injured cases from controls, 17 females have been reported to have lower CTS compared with males. 15 The latter would be consistent with females being more likely to demonstrate combined motion patterns of knee valgus and external rotation during landing (whereas males more often demonstrate varus and internal rotation), $\underline{4} \underline{32}$ combined motions more commonly associated with the ACL injury mechanism in females. 2,26 , 36 However, our data indicate that coupled motions of knee valgus and external rotation were more pronounced with higher MTS:LTS ratios. Although females have been reported to have greater MTS and LTS inclinations than do males, $\underline{15}, \underline{20}$ sex comparisons for MTS:LTS ratios have not been reported. Further research is needed to examine these combined slope characteristics in females and males, as well as examine their independent and interactive associations with hip and knee joint biomechanics during impact activities of increasing difficulty (eg, those performed on a single leg or that produce unbalanced multiplanar knee loads) and ultimately determine whether these altered biomechanics affect the threshold for ACL injury risk.

Although tibial geometry explained a significant amount of the variance in hip $\left(R^{2}=0.41-0.50\right)$ and knee $\left(R^{2}=0.21-0.59\right)$ kinematics, it cannot be determined whether the observed associations with kinematics are the direct result of alterations in knee joint geometry or whether knee joint geometry is acting as a surrogate for other concomitant functional adaptations (joint positioning) or structural factors not accounted for in the current study. For example, the finding that greater CTS and LTS predicted greater hip internal rotation at initial contact may be due to the body adopting a movement strategy at the hip to account for the greater tibial slopes at the knee or alternatively that knee joint geometry is acting as a surrogate for other related structural adaptations at the hip. It should also be noted that considerable variance remains unexplained by knee joint geometry and other lower extremity anatomic factors (eg, joint laxity, lower extremity alignment, lower extremity muscle strength), and their interactions may further influence hip and 
knee joint biomechanics during landing (as well as the relationship between knee joint geometry and landing biomechanics). A multifactorial investigation in a much larger cohort of participants is needed to fully understand the combined contributions of one's anatomy to his or her landing biomechanics.

Because of the larger study from which these data were derived, females were delimited to those with no history of knee ligament injury. As such, these participants and findings may not adequately represent the intersubject differences in the general female population that includes those at risk for ACL injury. We also acknowledge the limitations of using skin-based markers in assessing dynamic hip and knee joint function.2. 39 Although skin-based markers are commonly used in biomechanical studies assessing high-impact activities, 11,2433 it is well accepted that there is considerable variability associated with these measures. It is important to note, however, that this random variability only makes it more difficult to identify meaningful associations between 2 variables. In particular, the greater transverse plane hip and knee motion variability (see Appendix Table 1, available online) may have limited our ability to identify stronger associations with tibial geometry. New technologies such as biplane fluoroscopy $\underline{49}$ may allow for more precise examinations of these relationships during dynamic activities in the future.

\section{ACKNOWLEDGMENTS}

We thank Drs Anh-Dung Nguyen and Melissa Montgomery for their assistance with data collection.

\section{FOOTNOTES}

One or more of the authors has declared the following potential conflict of interest or source of funding: This study was supported in part by NIH-NIAMS Grant No. R01 AR053172.

\section{REFERENCES}

1. Arnold JA, Coker TP, Heaton LM, Park JP, Harris WD. Natural history of anterior cruciate tears. Am J Sports Med. 1979;7(6):305-313.

2. Benoit DL, Ramsey DK, Lamontagne M, Xu L, Wretenberg P, Renstrom P. Effect of skin movement artifact on knee kinematics during gait and cutting motions measured in vivo. Gait Posture. 2006;24:152-164.

3. Brandon ML, Haynes PT, Bonamo JR, et al. The association between posterior-inferior tibial slope and anterior cruciate ligament insufficiency. Arthroscopy. 2006;22(8):894-899.

4. Chappell JD, Herman DC, Knight BS, Kirkendall DT, Garrett WE, Yu B. Effect of fatigue on knee kinetics and kinematics in stop-jump tasks. Am J Sports Med. 2005;33(7):1022-1029.

5. Dejour H, Bonnin M. Tibial translation after anterior cruciate ligament rupture: two radiological tests compared. J Bone Joint Surg Br. 1994;76:745-749. 
6. Dempsey AR, Lloyd DG, Elliott BC, Steele JR, Munro BJ. Changing sidestep cutting technique reduces knee valgus loading. Am J Sports Med. 2009;37(11):2194-2200.

7. Ebstrup JF, Bojsen-Moller F. Anterior cruciate ligament injury in indoor ball games. Scand J Med Sci Sports. 2000;10:114-116.

8. Faul F, Erdfelder E, Lang AG, Buchner A. G*Power 3: a flexible statistical power analysis program for the social, behavioral, and biomedical sciences. Behav Res Methods. 2007;39(2):175-191.

9. Ferber R, Davis IM, Williams DS III. Gender differences in lower extremity mechanics during running. Clin Biomech. 2003;18(4):350-357.

10. Ford KR, Myer GD, Hewett TE. Valgus knee motion during landing in high school female and male basketball players. Med Sci Sports Exerc. 2003;35(10):1745-1750.

11. Ford KR, Myer GD, Smith RL, et al. A comparison of dynamic coronal plane excursion between matched male and female athletes when performing single leg landings. Clin Biomech. 2006;21:33-40.

12. Gagnon D, Gagnon M. The influence of dynamic factors on triaxial net muscular moments at the L5/S1 joint during asymmetrical lifting and lowering. J Biomech. 1992;25:891-901.

13. Giffin JR, Vogrin TM, Zantop T, Woo SL, Harner CD. Effects of increasing tibial slope on the biomechanics of the knee. Am J Sports Med. 2004;32(2):376-382.

14. Griffin LY, Albohm MJ, Arendt EA, et al. Understanding and preventing noncontact anterior cruciate ligament injuries: a review of the Hunt Valley II meeting, January 2005. Am J Sports Med. 2006;34(9):1512-1532.

15. Hashemi J, Chandrashekar N, Gill B, et al. The geometry of the tibial plateau and its influence on the biomechanics of the tibiofemoral joint. J Bone Joint Surg Am. 2008;90(12):2724-2734.

16. Hashemi J, Chandrashekar N, Gill B, et al. The geometry of the tibial plateau and tibiofemoral kinematics: a biomechanical analysis. Presented at: Annual Meeting of the American Society of Biomechanics; August 26-29, 2009; State College, PA. http://www.asbweb.org/conferences/2009/pdf/1131.pdf

17. Hashemi J, Chandrashekar N, Mansouri H, et al. Shallow medial tibial plateau and steep medial and lateral tibial slopes: new risk factors for anterior cruciate ligament injuries. Am J Sports Med. 2010;38(1):54-62.

18. Hewett TE, Ford KR, Myer GD, Wanstrath K, Scheper M. Gender differences in hip adduction motion and torque during a single-leg agility maneuver. J Orthop Res. 2006;24:416421. 
19. Hewett TE, Myer GD, Ford KR, et al. Biomechanical measures of neuromuscular control and valgus loading of the knee predict anterior cruciate ligament injury risk in female athletes: a prospective study. Am J Sports Med. 2005;33(4):492-501.

20. Hudek R, Fuchs B, Regenfelder F, Koch PP. Is noncontact ACL injury associated with the posterior tibial and meniscal slope? Clin Orthop Relat Res. 2011;469(8):2377-2384.

21. Imwalle L, Myer GD, Ford KR, Hewett TE. Relationship between hip and knee kinematics in athletic women during cutting maneuvers: a possible link to non-contact anterior cruciate ligament injury and prevention. J Strength Cond Res. 2009;23(8):2223-2230.

22. Johal P, Williams A, Wragg P, Hunt D, Gedroyc W. Tibio-femoral movement in the living knee: a study of weight bearing and non-weight bearing knee kinematics using 'interventional' MRI. J Biomech. 2005;38:269-276.

23. Kadaba MP, Ramakrishnan HK, Wootten ME, et al. Repeatability of kinematic, kinetic, and electromyographic data in normal adult gait. J Orthop Res. 1989;7:849-860.

24. Kernozek TW, Torry MR, Van Hoof H, Cowley H, Tanner S. Gender differences in frontal and sagittal plane biomechanics during drop landings. Med Sci Sports Exerc. 2005;37(6):10031012.

25. Khan MS, Seon JK, Song EK. Risk factors for anterior cruciate ligament injury: assessment of tibial plateau anatomic variables on conventional MRI using a new combined method. Int Orthop. 2011;35(8):1251-1256.

26. Krosshaug T, Nakamae A, Boden BP, et al. Mechanisms of anterior cruciate ligament injury in basketball: video analysis of 39 cases. Am J Sports Med. 2007;35(3):359-367.

27. Leardini A, Cappozzo A, Catani F, et al. Validation of a functional method for the estimation of hip joint centre location. J Biomech. 1999;32:33-103.

28. Madigan ML, Pidcoe PE. Changes in landing biomechanics during a fatiguing landing activity. J Electromyogr Kinesiol. 2003;13:491-498.

29. Malinzak RA, Colby SM, Kirkendall DT, Yu B, Garrett WE. A comparison of knee joint motion patterns between men and women in selected athletic tasks. Clin Biomech. 2001;16:438445.

30. Matsuda S, Miura H, Nagamine R, et al. Posterior tibial slope in the normal and varus knee. Am J Knee Surg. 1999;12:165-168.

31. McLean SG, Fellin R, Suedekum N, et al. Impact of fatigue on gender-based high-risk landing strategies. Med Sci Sports Exerc. 2007;39(3):502-514. 
32. McLean SG, Lipfert SW, Van den Bogert AJ. Effect of gender and defensive opponent on the biomechanics of sidestep cutting. Med Sci Sports Exerc. 2004;36(6):1008-1016.

33. McLean SG, Lucey SM, Rohrer S, Brandon C. Knee joint anatomy predicts high-risk in vivo dynamic landing knee biomechanics. Clin Biomech. 2010;25(8):781-788.

34. Meister K, Talley MC, Horodyski MB, et al. Caudal slope of the tibia and its relationship to noncontact injuries to the ACL. Am J Knee Surg. 1998;11:217-219.

35. Myer GD, Ford KR, Divine JG, et al. Longitudinal assessment of noncontact anterior cruciate ligament injury risk factors during maturation in a female athlete: a case control. J Athl Train. 2009;44(1):101-109.

36. Olsen OE, Myklebust G, Engebretsen L, Bahr R. Injury mechanisms for anterior cruciate ligament injuries in team handball: a systematic video analysis. Am J Sports Med. 2004;32(4):1002-1012.

37. Padua DA, Marshall SW, Beutler A, et al. The Landing Error Scoring System (LESS) prospectively identifies ACL injury. J Athl Train. 2010;45(5):539.

38. Portney LG, Watkins MP. Foundations of Clinical Research: Applications to Practice. 2nd ed. Upper Saddle River, NJ: Prentice Hall; 2000.

39. Reinschmidt C, van den Bogert AJ, Lundberg A, et al. Tibiofemoral and tibiocalcaneal motion during walking: external vs. skeletal markers. Gait Posture. 1997;6(2):98-109.

40. Renstrom P, Ljungqvist A, Arendt E, et al. Non-contact ACL injuries in female athletes: an International Olympic Committee current concepts statement. Br J Sports Med. 2008;42(6):394412.

41. Shimokochi Y, Shultz SJ. Mechanisms of noncontact anterior cruciate ligament injuries. J Athl Train. 2008;43(4):396-408.

42. Shultz SJ, Nguyen AD, Leonard MD, et al. Thigh strength and activation as predictors of knee biomechanics during a drop jump task. Med Sci Sports Exerc. 2009;41(4):857-866.

43. Shultz SJ, Schmitz RJ. Effects of transverse and frontal plane knee laxity on hip and knee neuromechanics during drop landings. Am J Sports Med. 2009;37(9):1821-1830.

44. Shultz SJ, Schmitz RJ, Kong Y, et al. Cyclic variations in multi-planar knee laxity influence landing biomechanics. Med Sci Sports Exerc. 2012;44(5):900-909.

45. Shultz SJ, Schmitz RJ, Nguyen A, Levine BJ. Joint laxity is related to lower extremity energetics during a drop jump landing. Med Sci Sports Exerc. 2010;42(4):771-780. 
46. Simon RA, Everhart JS, Nagaraja HN, Chaudhari AM. A case-control study of anterior cruciate ligament volume, tibial plateau slopes and intercondylar notch dimensions in ACLinjured knees. J Biomech. 2010;43:1702-1707.

47. Stijak L, Herzog RF, Schai P. Is there an influence of the tibial slope of the lateral condyle on the ACL lesion? A case-control study. Knee Surg Sports Traumatol Arthrosc. 2008;16(2):112117.

48. Todd MS, Lalliss S, Garcia S, DeBerardino TM, Cameron KL. The relationship between posterior tibial slope and anterior cruciate ligament injuries. Am J Sports Med. 2010;38(1):6367.

49. Torry MR, Shelburne KB, Peterson DS, et al. Knee kinematic profiles during drop landings: a biplane fluoroscopy study. Med Sci Sports Exerc. 2011;43(3):533-541.

50. Vyas S, van Eck CF, Vyas N, Fu FH, Otsuka NY. Increased medial tibial slope in teenage pediatric population with open physes and anterior cruciate ligament injuries. Knee Surg Sports Traumatol Arthrosc. 2010;19(3):372-377. 